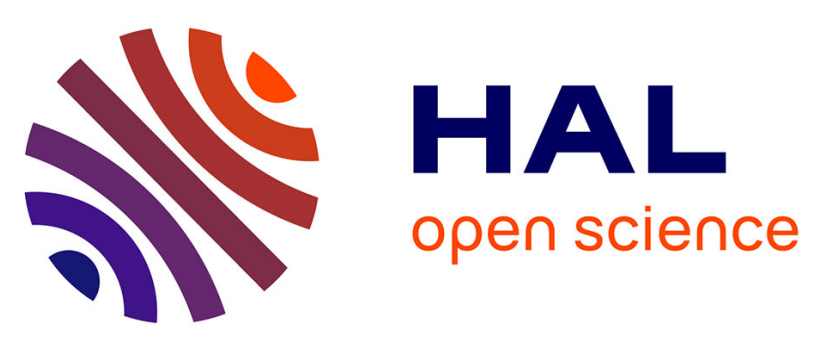

\title{
The protective effect of female gender on the development of albuminuria in a polygenetic rat model is further enhanced by replacement of a major autosomal QTL
}

Angela Schulz, Maria Schlesener, Judith Weiss, Jonna Hänsch, Norbert Wendt, Peter Kossmehl, Daniela Grimm, Roland Vetter, Reinhold Kreutz

\section{To cite this version:}

Angela Schulz, Maria Schlesener, Judith Weiss, Jonna Hänsch, Norbert Wendt, et al.. The protective effect of female gender on the development of albuminuria in a polygenetic rat model is further enhanced by replacement of a major autosomal QTL. Clinical Science, 2007, 114 (4), pp.305-311. 10.1042/CS20070300 . hal-00479398

\section{HAL Id: hal-00479398 https://hal.science/hal-00479398}

Submitted on 30 Apr 2010

HAL is a multi-disciplinary open access archive for the deposit and dissemination of scientific research documents, whether they are published or not. The documents may come from teaching and research institutions in France or abroad, or from public or private research centers.
L'archive ouverte pluridisciplinaire HAL, est destinée au dépôt et à la diffusion de documents scientifiques de niveau recherche, publiés ou non, émanant des établissements d'enseignement et de recherche français ou étrangers, des laboratoires publics ou privés. 


\section{The protective effect of female gender on the development of albuminuria in a polygenetic rat model is further enhanced by replacement of a major autosomal QTL}

Angela Schulz ${ }^{1}$, Maria Schlesener ${ }^{1}$, Judith Weiss ${ }^{1}$, Jonna Hänsch ${ }^{1}$, Norbert Wendt ${ }^{1}$, Peter Kossmehl $^{1}$, Daniela Grimm ${ }^{1}$, Roland Vetter ${ }^{1}$, Reinhold Kreutz ${ }^{1}$

${ }^{1}$ Institut für Klinische Pharmakologie und Toxikologie, Campus Benjamin Franklin, Charité - Universitätsmedizin Berlin, Berlin, Germany

Running head: gender and albuminuria in the MWF rat

\section{Correspondence to:}

Reinhold Kreutz, MD, FAHA

Department of Clinical Pharmacology and Toxicology

Charité - Universitätsmedizin Berlin

Campus Benjamin Franklin

Hindenburgdamm 30

12200 Berlin

Germany

Tel: $+49-30-8445-2280$

Fax: $+49-30-8445-4482$

e-mail: reinhold.kreutz@charite.de

Key words: gender, genetics, kidney, albuminuria, hypertension, rat 


\begin{abstract}
Clinical and experimental studies indicate that the progression of renal disease is faster in males than females. These observations are corroborated by a sexual dimorphism observed in the polygenetic Munich Wistar Frömter (MWF) rat model. The age dependent spontaneous progression of increased urinary albumin excretion (UAE) in male MWF is influenced by multiple quantitative trait loci (QTL). In contrast, female MWF develop only a slight increase in UAE, while the role of genetic factors for this phenotype is unknown. Here we show that compared to resistant spontaneously hypertensive rats (SHR) both male and female MWF develop a significant increase in UAE at 24 weeks of age ( $<<0.0001$, respectively), although blood pressures are lower compared to SHR $(\mathrm{p}<0.0001)$. UAE is significantly higher in male (7fold) compared to female MWF $(162.6 \pm 15.9$ vs. $24.0 \pm 5.5 \mathrm{mg} / 24 \mathrm{~h}, \mathrm{p}<0.0001)$ and only male MWF develop significant glomerulosclerosis and tubulointerstitial damage in the kidney $(p<0.0001)$. To test the role of genetic factors for the development of low grade albuminuria in female MWF we analyzed the role of a major UAE QTL on rat chromosome 6. To this end we analyzed a consomic MWF- $6^{\mathrm{SHR}}$ strain in which chromosome 6 from SHR was introgressed into the MWF background. Time course analysis of UAE in females indicated that the mild increase of UAE in MWF was fully suppressed by exchange of rat chromosome 6. Thus, taken together with previous studies in males we show that RNO6 protects against the increase of albuminuria with age in both female and male MWF.
\end{abstract}




\section{Introduction}

Currently, it is becoming increasingly evident that the development, manifestation and progression of cardiovascular and renal diseases differ between women and men [5]. With regard to renal disease a large body of evidence obtained in various animal models indicated that the progression of renal injury and failure is more pronounced in males compared to females [5;19]. A previous meta-analysis involving 11,345 patients with nondiabetic renal disease confirmed these experimental observations by showing that progression of chronic renal disease is slower in female than in male patients [20]. However, another meta-analysis enrolling a significant smaller number of only 1,860 patients suggested that the rate of renal disease progression may be even faster in women compared to men if the analysis accounted for other factors that are associated with a faster rate of progression [12]. In diabetic nephropathy, i.e. the major single cause of end-stage renal disease, it remains unclear whether gender status affects the progression of the disease [2;5;31].

In addition to gender, experimental and clinical studies carried out in the last decade documented a role for genetic factors contributing to the development of both diabetic and nondiabetic renal disease [26;29]. An increase of urinary albumin excretion (UAE) is an important predictor for the development of chronic nephropathy and already a slight increase of UAE represents an independent risk factor for cardiovascular events and mortality not only in arterial hypertension and diabetes but also in the general population [4;29]. Recently, several quantitative trait loci (QTL) have been identified by genome-wide linkage analyses for UAE in patients with diabetes or hypertension and in the general population $[8 ; 9 ; 14]$ and in several hypertensive genetic rat models $[3 ; 10 ; 22 ; 27 ; 28 ; 30 ; 33]$. In accordance with other rat models of renal damage the Munich Wistar Frömter rat (MWF) exhibits a marked sexual dimorphism regarding the progression of renal injury [5;7;19]. Thus, male MWF rats exhibit spontaneous hypertension and increased UAE leading to overt proteinuria and progressive glomerulosclerosis with age [7]. In contrast, female rats develop lower blood pressures, only mild albuminuria and are protected from glomerulosclerosis [7;13;24]. We therefore focussed in our previous genetic studies aiming to dissect the genetic basis of UAE in the MWF strain only on male animals [27-29]. We recently demonstrated that although albuminuria in male MWF is determined by a complex interplay of several quantitative trait loci (QTL) genetic exchange of one major locus on rat chromosome 6 (RNO6) leads to marked suppression of early onset albuminuria and subsequent renal damage in male animals [29]. This was achieved by targeted breeding and characterization of consomic MWF- ${ }^{\text {SHR }}$ rats in which RNO6 from the albuminuria resistant spontaneously hypertensive rat (SHR) [22;25] was 
introgressed into the isogenic background of MWF. However, the relevance of genetic factors for the modulation of mild albuminuria observed in female MWF animals has not been studied so far. We therefore asked the question whether genetic factors, i.e. QTL on RNO6, are also capable of modulating low grade albuminuria in female MWF that are protected from overt renal disease by their gender status.

\section{Methods}

\section{Animals and experimental groups}

All animals from MWF/Rkb, SHR/Rkb and our recently generated consomic MWF- ${ }^{\text {SHR }}$ strain [29] were obtained from our colonies (laboratory code $R k b$ ) at the Charité, Campus Benjamin Franklin, Germany. The consomic strain MWF- $6^{\text {SHR }}$ was derived from MWF/Rkb and $\mathrm{SHR} / R k b$ and was generated by sequential marker assisted backcrossing transferring the whole RNO6 from SHR into the MWF background as recently described [29]. Rats were grouped under conditions of regular $12 \mathrm{~h}$ diurnal cycles using an automated light switching device and climate-controlled conditions at a room temperature of $22^{\circ} \mathrm{C}$. The rats were fed a normal diet containing $0.2 \% \mathrm{NaCl}$ and had free access to food and water.

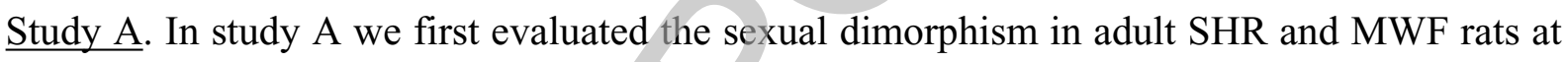
24 weeks of age. For this we selected male and female SHR (n=10 each) and MWF rats $(\mathrm{n}=18-19$ each) and studied systolic blood pressure (SBP), urinary albumin excretion (UAE) and performed renal histology analysis by determination of glomerulosclerosis and tubulointerstitial damage indices.

Study B. The aim of study B was to evaluate the role of RNO6 for the development of albuminuria and renal damage in female MWF rats. We therefore compared female consomic MWF-6 ${ }^{\text {SHR }}(n=20)$ rats with female MWF $(n=18)$ and female SHR $(n=10)$ with low UAE. In these animals we performed in accordance with previous studies [27-29] time-course analysis for $\mathrm{UAE}$ at $8,14,18$, and 24 weeks of age. In addition, we determined body and organ weights of heart and kidney, plasma concentrations of creatinine and urea, creatinine clearance, renal structural changes and renal collagen III mRNA expression.

In study $A$ and $B$ animals were killed after measuring UAE and systolic blood pressure at the age of 24 week.

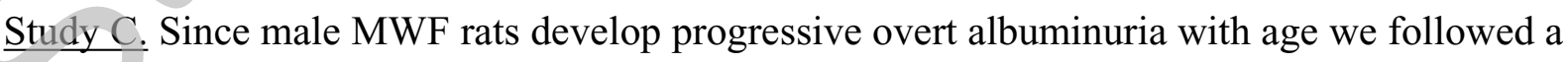
subgroup of female MWF and consomic MWF- $6^{\text {SHR }}$ animals ( $\mathrm{n}=8$, each) from study B to 
investigate the development of UAE in older female animals at 32 weeks. We compared the results with the data obtained in age-matched male animals analyzed in a recent report [29].

\section{Laboratory measurements}

For urine analysis animals were placed in metabolic cages for 2 days. The first day was used for adaptation and urine was collected for the last $24 \mathrm{~h}$ for biochemistry analysis. UAE as an indicator for renal damage was measured by a rat-specific ELISA-technique as previously described [13].

Systolic blood pressure was measured at 24 weeks of age by a noninvasive tail-cuff method in awake animals using a computer-assisted oscillatory detection device (TSE, Bad Homburg, Germany) as previously described [13]. These measurements involved 2 training sessions on 2 days followed by up to 18 (minimum 12) recordings in awake rats on 3 consecutive days was performed as previously reported [13]. Blood was drawn from the aorta for the determination of serum creatinine and urea with standard methods. Both kidneys and the heart were excised. The body, total kidney and heart weights were determined, respectively. For light microscopy evaluation the left kidney was fixed and embedded in paraffin for histology studies including determination of glomerulosclerosis index (GSI), tubulointerstitial damage index (TDI), and quantification of renal interstitial fibrosis (RIF) as reported [27].

\section{RNA analysis}

RNA was isolated from kidneys by the TRIzol reagent (Invitrogen, Karlsruhe, Germany) according to the manufacturer's instructions and was resuspended in DEPC-treated $\mathrm{H}_{2} \mathrm{O}$. First strand cDNA synthesis was carried out on $2 \mu \mathrm{g}$ of total RNA in a $20 \mu 1$ reaction using the First Strand cDNA Synthesis Kit (Fermentas Life Sciences, St. Leon-Rot, Germany) following the manufacturer's recommendations. To quantify mRNA expression of collagen III in kidney we employed a real-time quantitative RT (“TaqMan") PCR method as reported [29]. To normalize our expression data we used porphobilinogen deaminase (PBGD) as a housekeeping gene (GenBank accession no. X06827) as previously reported [29].

\section{Statistical analysis}

Statistical analysis was performed using two-way ANOVA followed by Bonferroni's adjustment and by non-parametric Mann-Whitney U-test. Data are presented as means \pm SEM and $\mathrm{p}$-values $<0.05$ were considered significant. 


\section{Results}

Study A. Evaluation of sexual dimorphism in adult SHR and MWF rats.

At the age of 24 weeks systolic blood pressure values were significantly lower in both adult male and female parental MWF rats compared to male and female SHR rats $(p<0.0001$; Figure 1A). A sexual dimorphism with higher systolic blood pressure in males compared to females ( $<<0.01$, respectively) was observed in SHR, whereas the blood pressure difference in MWF animals with somewhat lower values in females were not significant. In contrast to blood pressures UAE values are overall markedly elevated in MWF compared to SHR (Figure $1 \mathrm{~B}, \mathrm{p}<0.0001)$. Both male and female SHR animals demonstrated comparable low UAE values of $\sim 2 \mathrm{mg} / 24 \mathrm{~h}$, while MWF animals exhibited a striking sexual dimorphism with $\sim 7$ fold higher values in male $(162.6 \pm 15.9 \mathrm{mg} / 24 \mathrm{~h})$ compared to female $(24.0 \pm 5.5 \mathrm{mg} / 24 \mathrm{~h})$ animals (Figure 1B, $\mathrm{p}=0.0003$ ). Analysis of structural changes in the kidney of these animals at 24 weeks of age demonstrated a significant increase of GSI and TDI, i.e. glomerular and tubulointerstitial damage, in male MWF rats compared to female MWF and SHR animals of both sex (Figure 2, $\mathrm{p}<0.0001$, respectively). Representative pictures of this histology analysis performed after PAS staining are shown in Figure 3.

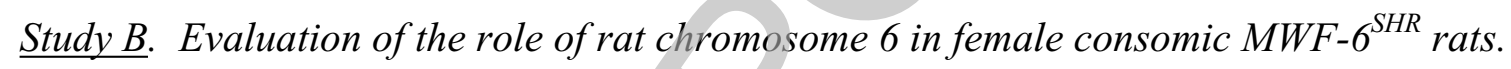

Time-course analysis for UAE between week 8 and 24 in female consomic MWF- $6^{\text {SHR }}$ as compared to parental MWF and SHR strains is shown in Figure 4. In contrast to SHR female MWF rats demonstrated already a significant increase of UAE at 8 weeks of age $(0.1 \pm 0.01 \mathrm{vs}$. $5.2 \pm 0.8 \mathrm{mg} / 24 \mathrm{~h}, \mathrm{p}<0.0001$, Figure 4). UAE progressed with age in female MWF and demonstrated mean values of $24.0 \mathrm{mg} / 24 \mathrm{~h}$ at 24 weeks. This increase was fully suppressed in female consomic animals at all time points investigated $(p<0.0001$, Figure 4); they only showed a slight increase with values up to $1.7 \mathrm{mg} / 24 \mathrm{~h}$ at 24 weeks of age. Overall, no significant differences in UAE levels between female consomic MWF- $6{ }^{\text {SHR }}$ and female SHR animals were observed. At 24 weeks of age analysis of variance revealed no significant differences for body weights and kidney weights between female rats of the three strains (Table 1). Determination of systolic blood pressure in consomic rats demonstrated values similar to female MWF, thus systolic blood pressure was lower in both strains compared to SHR $(\mathrm{p}<0.0001$, Table. 1). Replacement of RNO6 in the consomic strain resulted in lower serum urea concentrations compared to female parental MWF animals $(p<0.0001$, Table 1), while heart weight, serum creatinine and creatinine clearance at 24 weeks of age were not affected as summarized in Table 1. In addition, as expected from the data obtained for kidney 
histology analysis in female parental MWF and SHR rats, GSI and TDI were also low in female MWF- $6^{\text {SHR }}$ and not significantly different from female rats of the other two strains (not shown). However, the quantitative analysis of RIF in female MWF revealed a significant albeit slight increase in fibrosis compared to SHR $(\mathrm{p}=0.04)$, while consomic animals exhibited intermediate levels (Table 1). In addition, collagen III mRNA expression was significantly increased in female MWF compared to SHR animals ( $p=0.0002)$, whereas consomic animals demonstrated significantly lower expression levels compared to MWF $(p=0.04)$ and were not statistically different from SHR (Table 1).

$\underline{\text { Study } C}$. Role of rat chromosome 6 in aging female $M W F$ and comparison with male $M W F$ rats.

In contrast to old male animals, which develop according to our previous analysis mean UAE levels of up to $~ 355 \mathrm{mg} / 24 \mathrm{~h}$ (range 206-561 mg/24h) [29], female MWF demonstrated only UAE levels of $18.6 \pm 3.0 \mathrm{mg} / 24 \mathrm{~h}$ at 32 weeks of age. The latter values are comparable to the UAE rates observed in female MWF at 24 weeks of age (Figure 4). Thus, female MWF exhibit no significant progression of UAE between week 24 and 32. Despite the striking sexual dimorphism in absolute amounts of UAE between male and female MWF the relative suppression of UAE conferred by transfer of RNO6 in consomic MWF-6 ${ }^{\text {SHR }}$ animals appears similar in males and females as summarized in Figure 5 (about $85-95 \%$ reduction compared to parental MWF of the same sex).

\section{Discussion}

The MWF rat represents a valuable model to study the mechanisms involved in progressive glomerular injury and renal failure [15]. This inbred rat strain develops mild arterial hypertension, albuminuria, glomerulosclerosis and shows a reduction of nephron number [7;29]. In the present study we investigated the role of a previously identified important albuminuria QTL on RNO6 [28] for the development of albuminuria in female rats, which show in contrast to male rats only low grad albuminuria. Our time-course analysis of UAE in female consomic MWF- $6^{\text {SHR }}$ rats demonstrated that the mild increase of UAE observed in female MWF was fully suppressed by exchange of rat chromosome 6. Taken together with previous studies in males [29] we show that RNO6 protects against the increase of albuminuria with age in both female and male MWF.

The sexual dimorphism of renal disease phenotypes observed in this model reflects clinical findings in nondiabetic renal disease indicating that disease progression is lower in females 
than in males $[5 ; 19 ; 20 ; 31]$. However, the biological basis for this phenomenon is not fully understood. In MWF animals, both females and males share the same reduction in nephron number as demonstrated by Fassi et al. [7]. Thus, the lower UAE and the protection from overt proteinuria and structural damage of the kidney in female MWF rats are probably not attributable to differences in nephron development. Our previous studies in male MWF animals indicated that the increased UAE in MWF is a polygenetic trait that is influenced by up to 11 UAE QTL that are not linked to blood pressure [27;28]. Although the genetic studies in male MWF indicated that the increase in UAE is independent from blood pressure the possibility that the somewhat lower blood pressures observed in female MWF are contributing to the lower UAE levels in female animals could not be excluded.

On the other hand it appears possible that gender specific QTL effects [17] may explain in part the sexual dimorphism in UAE in the MWF strain. However, this question has not been addressed in previous QTL mapping studies, which included only male MWF animals because of their more pronounced phenotypes [27;28]. Similarly, QTL mapping studies for UAE phenotypes in the Dahl salt-sensitive were also restricted to male animals and led to the identification of multiple QTL in male rats only [10;22]. However, in a subsequent study by Garrett et al. the confirmation of UAE QTL in congenic strains derived from Dahl saltsensitive rats was reported to be similar in male and female animals although no data for female rats were shown [11].

As a result of the studies in male MWF we recently confirmed an important role of RNO6 for albuminuria development in male animals [29]. In the current study we show for the first time that genetic factors, i.e. QTL on RNO6, influence also the development of albuminuria in female MWF rats. Thus, transfer of RNO6 from SHR in the MWF background is protective in both sexes.

Comparative mapping analysis [32] between the $99 \%$ and $95 \%$ confidence intervals for placement of the albuminuria QTL on RNO6 with the human genome maps this region to human chromosome 14q23.1-14q31.3. This region contains 146 annotated genes. Currently, we are not able to give any indication which gene(s) within the RNO6 region is (are) responsible for onset of albuminuria in MWF. However, the data in both female and male MWF- $6^{\text {SHR }}$ validate this region and provide the rationale to further develop congenic rats carrying smaller intervals, thereby reducing the number of genes within the QTL for further investigation. 
It has been shown already that morphologic changes of the glomerular basement membrane or ultrastructure of the podocytes are not responsible for the development of albuminuria in the MWF model [16]. More recent data demonstrated that progressive proteinuria in male animals is associated with damage and loss of podocytes [15]. Since renal damage is only mild in female compared to male MWF animals no major differences in renal function and renal structural damage were detected in consomic animals as expected, except that serum urea and renal collagen III expression were significantly reduced compared to MWF parental animals.

Previously, Fassi et al. provided evidence for glomerular hyperfiltration in MWF animals by showing a twofold increase of single nephron glomerular filtration rate in strain [7]. In addition, they demonstrated that in comparison to normal Wistar rats both male and female MWF animals demonstrated a similar $\sim 50 \%$ reduction in total glomeruli number [7]. Consequently, in the latter study total glomerular filtration rate was not significantly different between sex-matched MWF and Wistar rats at an early adult age of 12-14 weeks. The data obtained in the current study indicate that renal function as determined by serum creatinine concentrations and creatinine-clearance is impaired in female MWF and consomic MWF- $6^{\text {SHR }}$ compared to SHR at 24 weeks of age. It is well established that renal function is progressively declining in aging male MWF rats with progressive proteinuria probably due to severe structural damage [23;29]. Although our current data would suggest that overall glomerular filtration rate is also lower in older female MWF compared to SHR it appears possible that single glomeruli of female MWF are hyperfiltrating, since we have recently shown that the total number of glomeruli in the MWF strain is also significantly reduced $(\sim-30 \%)$ when compared to the SHR strain [29]. On the other hand, our findings document that the lower creatinine-clearance in MWF and consomic animals can not be related to differences in albuminuria, which was similar between consomic animals and SHR, or to differences in renal structural damage, which was low and similar in females of all three strains. To obtain further insights into the renal function of female MWF and consomic animals would require additional studies investigating renal and glomerular hemodynamics in relation to total number of glomeruli in these animals and in comparison to female SHR using appropriate methods, e.g. inulin-clearance measurements for the determination of glomerular filtration rate [7]. Systolic blood pressures were similar in female MWF and consomic animals thus clearly ruling out a role of blood pressure differences as an explanation for the suppressed albuminuria in MWF- $6^{\mathrm{SHR}}$. 
It is well established that aging male animals of some strains of rats develop spontaneous proteinuria and glomerulosclerosis, while treatment with estrogens or orchiectomy protects against renal damage in older age [19]. In addition, investigations in various renal disease models indicated that progression of renal injury can be modulated by surgical or pharmacological change of the sex hormonal status in both male and female rats [19]. These data support the overall concept that androgens are deleterious and estrogens are protective for renal disease progression in experimental models. Moreover, gender-specific differences of important factors regulating renal function such as the renin-angiotensin-system $[5 ; 18 ; 21]$ and the renal nitric oxide synthase isoforms [6;34] have been implicated in the sexual dimorphism of renal disease progression. Previous studies performed in Munich Wistar rats, i.e. the genetic background of MWF animals, indicated that the presence of androgens in male rats rather than the absence of estrogens provide the risk factor for development of agedependent proteinuria and glomerular damage in this strain [1]. The current study demonstrates that early onset of albuminuria at 8 weeks of age in female MWF is comparable to the early increase of UAE in male rats [29]. Subsequently, female rats develop only a slight increase of UAE after maturation reaching a plateau between week 24 and 32, while males show a marked progression with age. The similar suppression of albuminuria in female and male rats induced by exchange of RNO6 QTL supports the conclusion that the same genetic mechanisms are responsible for the onset of albuminuria in both male and female MWF rats, while the sexual dimorphism is probably attributable to non-genetic factors such as the influence of sex hormones. Future studies may aim to characterize how changes in sex hormonal status affect the genotype-phenotype affects in albuminuria.

\section{Acknowledgements}

We acknowledge the contributions of Sabine Wunderlich and Claudia Plum for laboratory assistance and of Bettina Bublath for support in animal breeding. This study was supported by grants from the DFG KR1152-3-1. 


\section{References:}

1. Baylis,C. (1994) Age-dependent glomerular damage in the rat. Dissociation between glomerular injury and both glomerular hypertension and hypertrophy. Male gender as a primary risk factor. J Clin.Invest 94, 1823-1829.

2. Breyer,M.D. (2007) Diabetic nephropathy: introduction. Semin.Nephrol. 27, 129.

3. Brown,D.M., Provoost,A.P., Daly,M.J., Lander,E.S. and Jacob,H.J. (1996) Renal disease susceptibility and hypertension are under independent genetic control in the fawn-hooded rat. Nat Genet 12, 44-51.

4. De Jong,P.E. and Curhan,G.C. (2006) Screening, monitoring, and treatment of albuminuria: Public health perspectives. J Am Soc Nephrol 17, 2120-2126.

5. Denton,K. and Baylis,C. (2007) Physiological and molecular mechanisms governing sexual dimorphism of kidney, cardiac, and vascular function. Am.J.Physiol Regul.Integr.Comp Physiol. 292, R697-R699.

6. Erdely,A., Greenfeld,Z., Wagner,L. and Baylis,C. (2003) Sexual dimorphism in the aging kidney: Effects on injury and nitric oxide system. Kidney Int. 63, 1021-1026.

7. Fassi,A., Sangalli,F., Maffi,R. et al. (1998) Progressive glomerular injury in the MWF rat is predicted by inborn nephron deficit. J Am Soc Nephrol 9, 1399-1406.

8. Fox,C.S., Yang,Q., Guo,C.Y. et al. (2005) Genome-wide linkage analysis to urinary microalbuminuria in a community-based sample: the Framingham Heart Study. Kidney Int. 67, 70-74.

9. Freedman,B.I., Beck,S.R., Rich,S.S. et al. (2003) A genome-wide scan for urinary albumin excretion in hypertensive families. Hypertension 42, 291-296.

10. Garrett,M.R., Dene,H. and Rapp,J.P. (2003) Time-course genetic analysis of albuminuria in dahl saltsensitive rats on low-salt diet. J Am Soc Nephrol JID - 9013836 14, 1175-1187.

11. Garrett,M.R., Joe,B. and Yerga-Woolwine,S. (2006) Genetic linkage of urinary albumin excretion in Dahl salt-sensitive rats: influence of dietary salt and confirmation using congenic strains. Physiol Genomics 25, $39-49$.

12. Jafar,T.H., Schmid,C.H., Stark,P.C. et al. (2003) The rate of progression of renal disease may not be slower in women compared with men: a patient-level meta-analysis. Nephrol.Dial.Transplant. 18, 20472053.

13. Kreutz,R., Kovacevic,L., Schulz,A., Rothermund,L., Ketteler,M. and Paul,M. (2000) Effect of high NaCl diet on spontaneous hypertension in a genetic rat model with reduced nephron number. J Hypertens $\mathbf{1 8}$, 777-782. 
14. Krolewski,A.S., Poznik,G.D., Placha,G. et al. (2006) A genome-wide linkage scan for genes controlling variation in urinary albumin excretion in type II diabetes. Kidney Int. 69, 129-136.

15. Macconi,D., Bonomelli,M., Benigni,A. et al. (2006) Pathophysiologic implications of reduced podocyte number in a rat model of progressive glomerular injury. Am.J.Pathol. 168, 42-54.

16. Macconi,D., Ghilardi,M., Bonassi,M.E. et al. (2000) Effect of angiotensin-converting enzyme inhibition on glomerular basement membrane permeability and distribution of zonula occludens- 1 in MWF rats. J Am Soc Nephrol JID - 9013836 11, 477-489.

17. McBride,M.W., Carswell,H.V., Graham,D. et al. (2002) Genetic and gender determinants of cerebrovascular disease. Semin.Nephrol. 22, 127-134.

18. Miller,J.A., Cherney,D.Z., Duncan,J.A. et al. (2006) Gender differences in the renal response to reninangiotensin system blockade. J Am Soc Nephrol. 17, 2554-2560.

19. Neugarten,J. (2002) Gender and the progression of renal disease. J Am.Soc.Nephrol. 13, 2807-2809.

20. Neugarten,J., Acharya,A. and Silbiger,S.R. (2000) Effect of gender on the progression of nondiabetic renal disease: a meta-analysis. J Am.Soc.Nephrol. 11, 319-329.

21. Paul,M., Poyan,M.A. and Kreutz,R. (2006) Physiology of local renin-angiotensin systems. Physiol Rev. 86, 747-803.

22. Poyan,M.A., Siegel,A.K., Kossmehl,P. et al. (2003) Early onset albuminuria in Dahl rats is a polygenetic trait that is independent from salt loading. Physiol Genomics 14, 209-216.

23. Remuzzi,A., Gagliardini,E., Sangalli,F. et al. (2006) ACE inhibition reduces glomerulosclerosis and regenerates glomerular tissue in a model of progressive renal disease. Kidney Int.

24. Remuzzi,A., Puntorieri,S., Alfano,M. et al. (1992) Pathophysiologic implications of proteinuria in a rat model of progressive glomerular injury. Lab Invest 67, 572-579.

25. Rothermund,L., Luckert,S., Kossmehl,P., Paul,M. and Kreutz,R. (2001) Renal endothelin ET(A)/ET(B) receptor imbalance differentiates salt-sensitive from salt-resistant spontaneous hypertension. Hypertension 37, 275-280.

26. Satko,S.G., Sedor,J.R., Iyengar,S.K. and Freedman,B.I. (2007) Familial clustering of chronic kidney disease. Semin.Dial. 20, 229-236.

27. Schulz,A., Litfin,A., Kossmehl,P. and Kreutz,R. (2002) Genetic dissection of increased urinary albumin excretion in the munich wistar fromter rat. J Am Soc Nephrol 13, 2706-2714.

28. Schulz,A., Standke,D., Kovacevic,L. et al. (2003) A major gene locus links early onset albuminuria with renal interstitial fibrosis in the MWF rat with polygenetic albuminuria. J Am Soc Nephrol 14, 3081-3089. 
29. Schulz,A., Weiss,J., Schlesener,M. et al. (2007) Development of overt proteinuria in the Munich Wistar Fromter rat is suppressed by replacement of chromosome 6 in a consomic rat strain. J Am.Soc.Nephrol. 18, $113-121$.

30. Shiozawa,M., Provoost,A.P., van Dokkum,R.P., Majewski,R.R. and Jacob,H.J. (2000) Evidence of genegene interactions in the genetic susceptibility to renal impairment after unilateral nephrectomy. JAm Soc Nephrol JID - 9013836 11, 2068-2078.

31. Silbiger,S.R. and Neugarten,J. (2003) The role of gender in the progression of renal disease. Adv.Ren Replace.Ther. 10, 3-14.

32. Stoll,M., Kwitek-Black,A.E., Cowley,A.W.J. et al. (2000) New target regions for human hypertension via comparative genomics. Genome Res 10, 473-482.

33. Yagil,C., Sapojnikov,M., Wechsler,A., Korol,A. and Yagil,Y. (2006) Genetic dissection of proteinuria in the Sabra rat. Physiol Genomics.

34. Yamaleyeva,L.M., Gallagher,P.E., Vinsant,S. and Chappell,M.C. (2007) Discoordinate regulation of renal nitric oxide synthase isoforms in ovariectomized mRen2.Lewis rats. Am J Physiol Regul Integr Comp Physiol. 292, R819-R826. 


\section{Figure legends}

\section{Figure 1.}

Systolic blood pressure (SBP) in panel A and urinary albumin excretion (UAE) in panel B of female (open bars) and male (solid bars) spontaneously hypertensive rats (SHR) and Munich Wistar Frömter rats (MWF) at 24 weeks of age. ${ }^{*} \mathrm{P}<0.0001$ vs. SHR same gender; $\# \mathrm{P}<0.01$ vs. males same strain.

\section{Figure 2.}

Glomerulosclerosis index (GSI) in panel A and tubulointerstitial damage index (TDI) in panel B of female (open bars) and male (solid bars) spontaneously hypertensive rats (SHR) and Munich Wistar Frömter rats (MWF) at 24 weeks of age. ${ }^{*} \mathrm{P}<0.0001$ compared to other groups, respectively.

\section{Figure 3.}

Histopathology of the kidney in rat strains at 24 weeks of age, respectively. Paraffin sections were stained with periodic acid-Schiff reagent (PAS). Panels A, B, and C represent female rats, respectively, of spontaneously hypertensive rat (SHR) in A, Munich Wistar Frömter rat $(\mathrm{MWF})$ in B, and consomic MWF- $6^{\mathrm{SHR}}$ rat in C. Panels D, E, and F represent the corresponding male animals. All female animals (panels A, B, and C) and male SHR (panel D) demonstrate no severe abnormalities. In contrast, male MWF (panel E) show significant glomerulosclerosis in two glomeruli, tubulointerstitial infiltration (arrow), and tubular casts (*), while male consomic MWF- $6^{\mathrm{SHR}}$ (panel F) show intermediate glomerulosclerosis.

\section{Figure 4.}

Time course-analysis of urinary albumin excretion (UAE) of female spontaneously hypertensive rats (SHR) in white bars, consomic MWF- $6^{\text {SHR }}$ in grey bars, Munich Wistar Frömter rats (MWF) in black bars at 8, 14, 18, and 24 weeks of age. ${ }^{*} \mathrm{P}<0.0001$ compared to SHR and MWF-6 ${ }^{\text {SHR }}$, respectively.

\section{Figure 5.}

Urinary albumin excretion (UAE) in male and female consomic MWF-6 ${ }^{\text {SHR }}$ (grey bars) and Munich Wistar Frömter (MWF) rats (black bars) at 32 wk of age. ${ }^{*} \mathrm{P}<0.0001$ compared to 
consomic animals. The numbers over consomic animals (grey bars) indicate the percentage of UAE in relation to the parental MWF strain. 
Table 1. Overall characteristics of female parental MWF, SHR, and consomic MWF- $6^{\text {SHR }}$ rats at 24 weeks o.

\begin{tabular}{|c|c|c|c|c|c|c|c|}
\hline & SHR & MWF-6 ${ }^{\text {SHR }}$ & MWF & Overall & $\begin{array}{r}\text { P - A } \\
\text { MWF vs. } \\
\text { SHR } \Phi_{0}^{\circ} \\
\end{array}$ & $\begin{array}{l}\text { OVA } \\
\text { MWF YS. } \\
\text { MWF-6 }\end{array}$ & $\begin{array}{l}\text { SHR vs. } \\
\text { MWF-6 }\end{array}$ \\
\hline Body weight (g) & $208.0 \pm 5.5$ & $214.4 \pm 5.2$ & $207.2 \pm 1.2$ & 0.4 & & & \\
\hline Systolic blood pressure $(\mathrm{mmHg})$ & $157.1 \pm 2.9$ & $139.0 \pm 1.6$ & $138.5 \pm 1.2$ & $<0.0001$ & U & 1.0 & $<0.0001$ \\
\hline Kidney weight (g) & $1.28 \pm 0.05$ & $1.30 \pm 0.05$ & $1.20 \pm 0.03$ & 0.07 & $V$ & & \\
\hline Kidney weight/Body weight (g) & $6.15 \pm 0.16$ & $6.06 \pm 0.16$ & $5.78 \pm 0.13$ & 0.09 & $\frac{i}{u}$ & & \\
\hline Heart weight $(g)$ & $0.76 \pm 0.03$ & $0.66 \pm 0.03$ & $0.62 \pm 0.01$ & $<0.0001$ & $<0.001$ 㞬 & 1.0 & 0.02 \\
\hline Heart weight/Body weight $(\mathrm{mg} / \mathrm{g})$ & $3.67 \pm 0.10$ & $3.08 \pm 0.10$ & $2.98 \pm 0.02$ & $<0.0001$ & $<0.0001$ 으 & 1.0 & $<0.0001$ \\
\hline Serum creatinine $(\mu \mathrm{mol} / \mathrm{l})$ & $35.5 \pm 1.6$ & $42.3 \pm 1.1$ & $41.9 \pm 0.4$ & $<0.001$ & $<0.01 \stackrel{\text { L }}{\underline{z}}$ & 1.0 & 0.002 \\
\hline Serum urea $(\mathrm{mmol} / \mathrm{l})$ & $6.83 \pm 0.24$ & $6.88 \pm 0.24$ & $9.32 \pm 0.39$ & $<0.0001$ & $<0.0001 \frac{\varrho}{I}$ & $<0.0001$ & 1.0 \\
\hline Crea Cl $(\mathrm{ml} / \mathrm{min} \bullet 100 \mathrm{~g})$ & $0.47 \pm 0.05$ & $0.37=0.04$ & $0.38 \pm 0.02$ & 0.02 & 0.56 & 1.0 & 0.45 \\
\hline Renal interstitial fibrosis (\%) & $4.0 \pm 0.2$ & $4.5 \pm 0.1$ & $4.9 \pm 0.3$ & 0.05 & 0.04 & 0.92 & 0.35 \\
\hline Collagen III/PBGD (ratio) & $1.8 \pm 0.2$ & $2.9 \pm 0.3$ & $4.5 \pm 0.7$ & 0.0003 & 0.0002 & 0.04 & 0.16 \\
\hline
\end{tabular}

Crea Cl, creatinine clearance; MWF, Munich Wistar Frömter; SHR, spontaneously hypertensive rat; PBGD, porphobilinogen deaminase. 
Figure 1

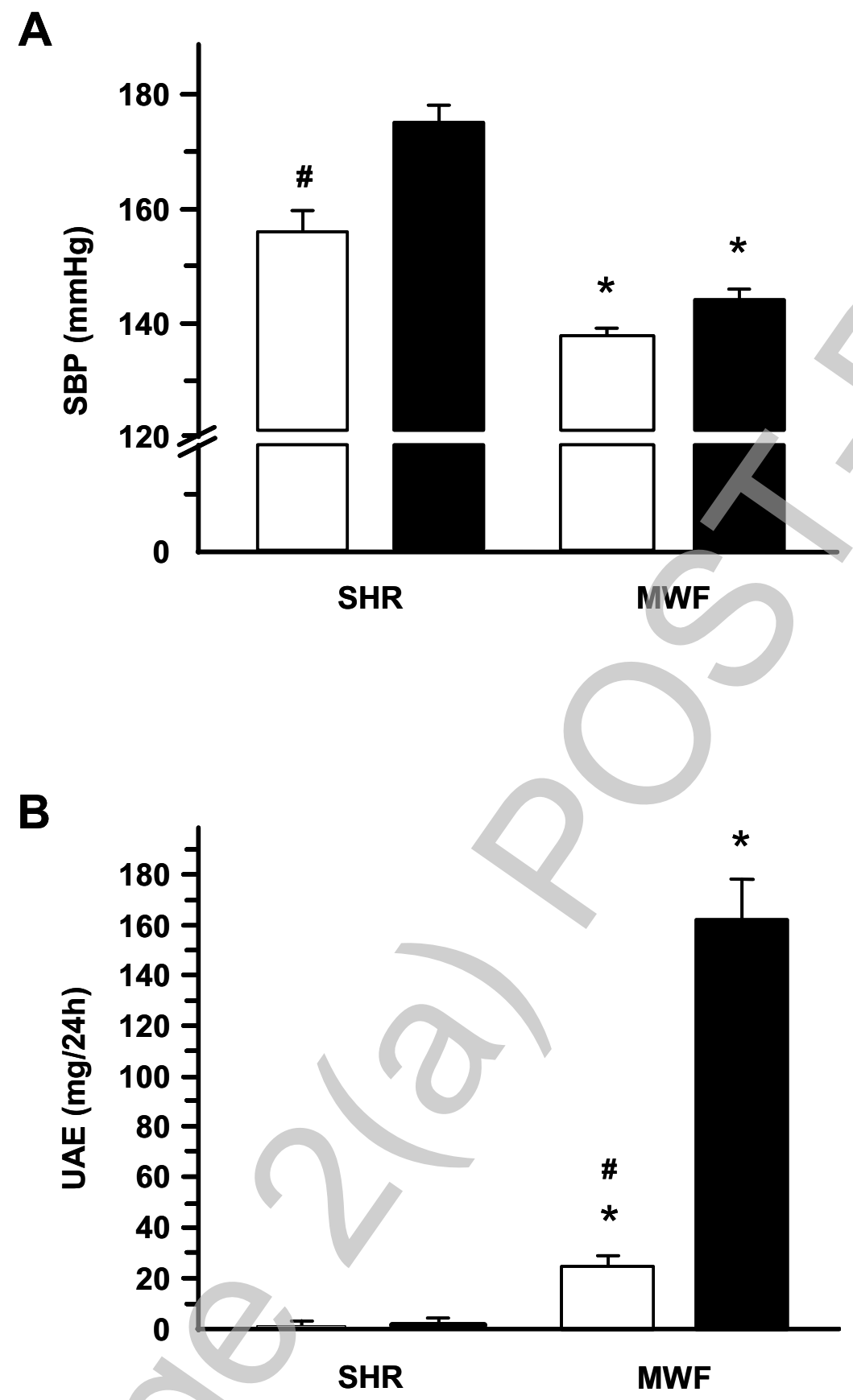


Figure 2

A

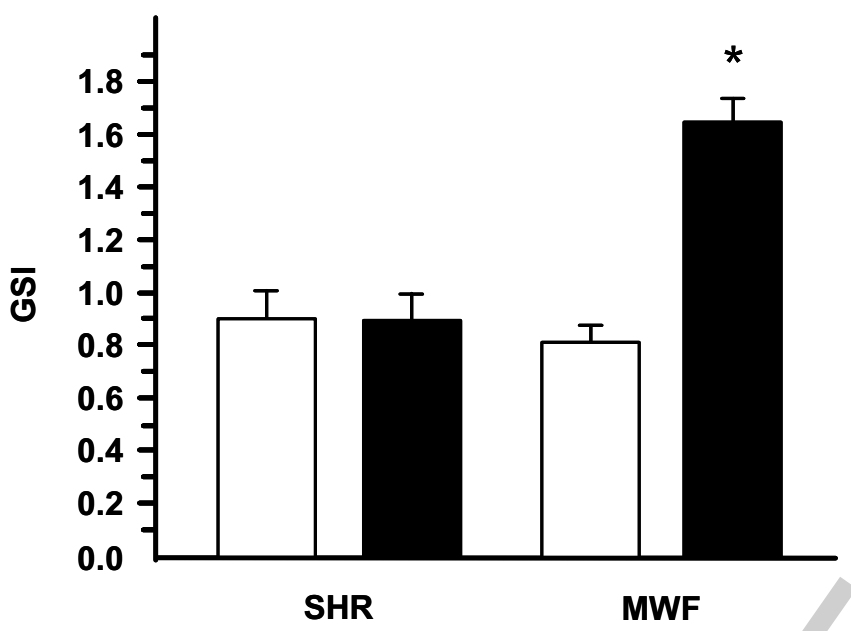

B

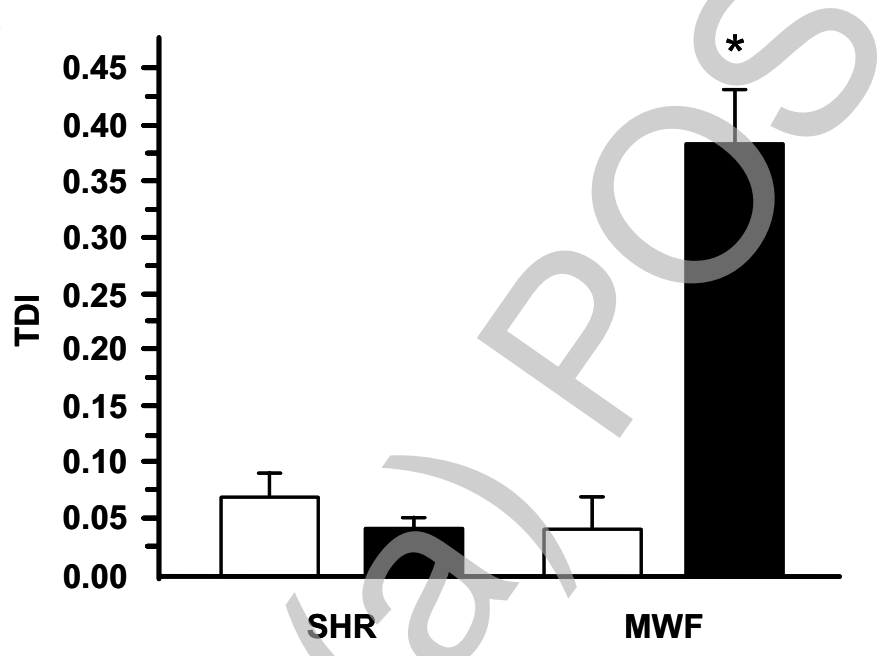


Figure 3

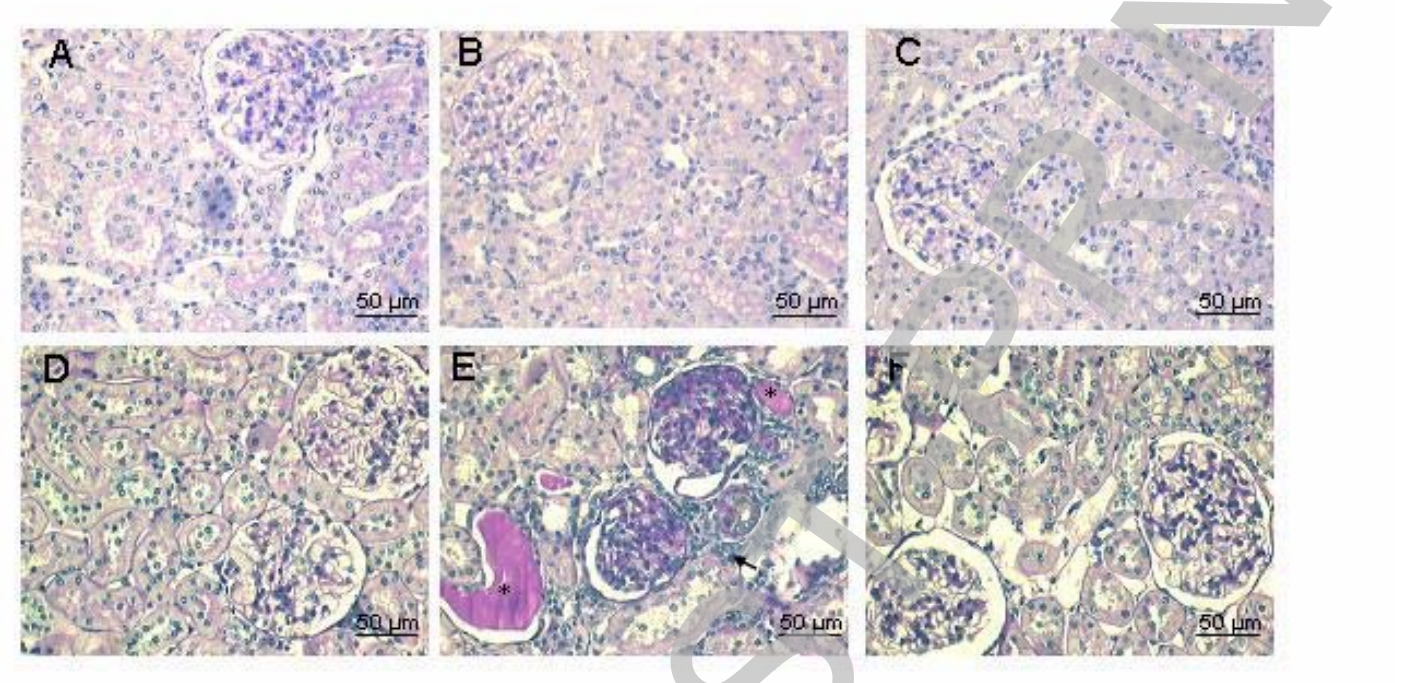


Figure 4

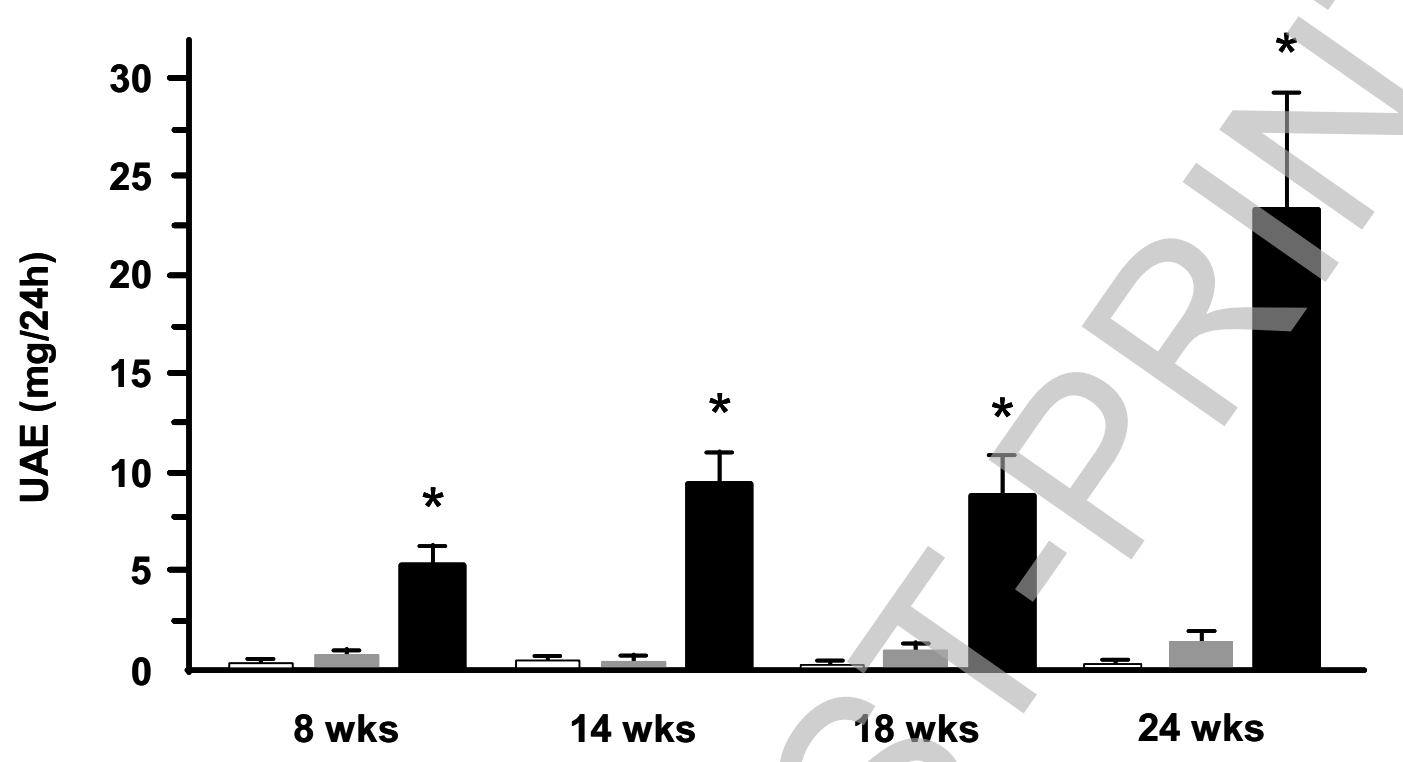


Figure 5

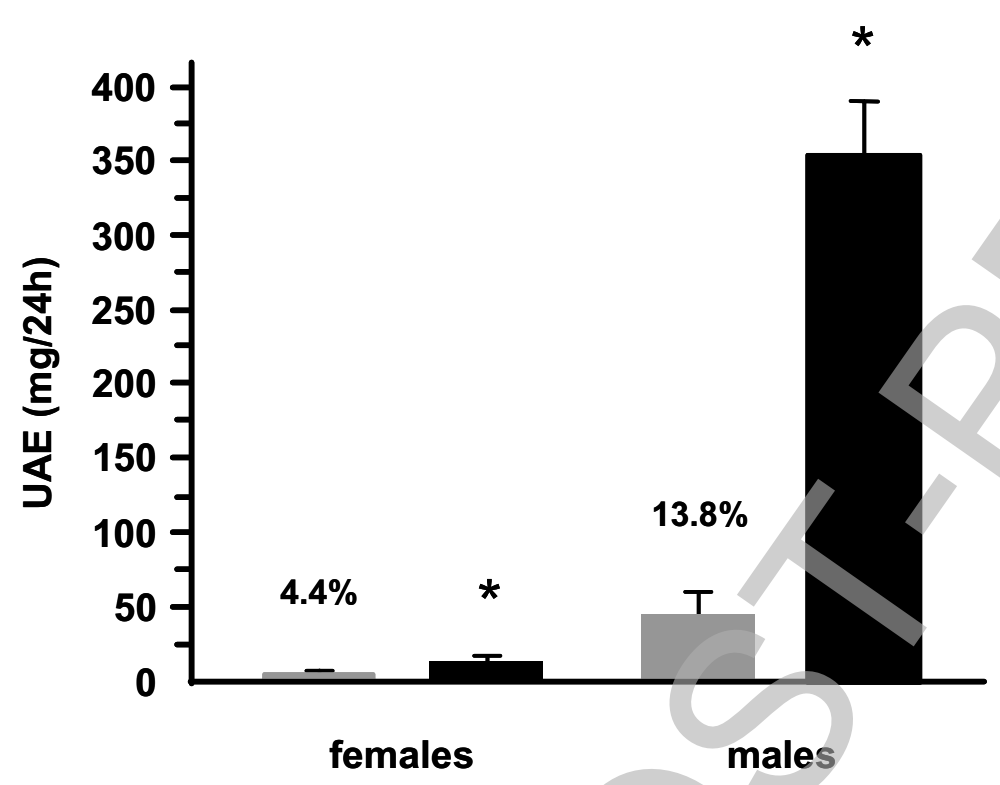

